Einführung zum Thema

Hautarzt 2020 $71: 919$

https://doi.org/10.1007/s00105-020-04718-9

(C) Springer Medizin Verlag GmbH, ein Teil von Springer Nature 2020

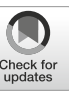

\author{
Klaus Fritz ${ }^{1,2} \cdot$ Carmen Salavastru ${ }^{3,4}$ \\ ${ }^{1}$ Hautärzte und Laserzentrum, Landau, Deutschland \\ ${ }^{2}$ Medizinische und Pharmazeutische Universität Carol Davila, Bukarest, Rumänien \\ ${ }^{3}$ Abteilung für pädiatrische Dermatologie, Colentina-Krankenhaus, Bukarest, Rumänien \\ ${ }^{4}$ Dermatologie, Colentina-Krankenhaus, Bukarest, Rumänien
}

\title{
Kurative Behandlung mit Lasern kann Erkrankungen der Haut mit Pigmentstörungen lindern
}

Lebensqualität aus. Daraus erklärt sich auch die hohe Nachfrage von Patienten nach wirksamen Methoden, um störende Pigmentierungen zu entfernen.

\section{》) Pigmentstörungen sind oft nicht lebensbedrohlich, wirken sich aber negativ auf die Lebensqualität aus} eher selten, ebenso die genetisch bedingten Veränderungen. Medikamente können Pigmentstörung als Nebenwirkung aufweisen, hormonelle Veränderungen und Einflüsse können zu gestört pigmentierter Haut führen, gelegentlich auch Kosmetika, physikalische Faktoren, UV(Ultraviolett)-Licht, Entzündungen oder immunologische Erkrankungen.

Dieses Leitthemenheft beschäftigt sich allerdings weniger mit der Differenzialdiagnostik und Ursachenklärung, sondern mit den oft begrenzten therapeutischen Möglichkeiten von Lokalbehandlungen bis zur Lasertherapie. International, v. a. in Ländern mit dunkleren Hauttypen, ist das Thema Pigmentstörungen von hoher Bedeutung, führen diese doch zu Auffälligkeiten, die individuell Stigmatisierung und Ausgrenzung nach sich ziehen können. Das wiederum wirkt sich negativ auf die Psyche aus. Betroffene können ein verringertes Selbstwertgefühl und Minderwertigkeitskomplexe entwickeln, sogar psychische Verstimmungen bis zur Depression. Meist sind diese Pigmentstörungen nicht lebensbedrohlich, wirken sich aber sehr negativ auf die
Leider sind nicht viele Methoden verfügbar, die erfolgreich und nachhaltig wirken. Dieses Heft soll dazu dienen, diese Methoden mit ihren Möglichkeiten und Limitierungen darzustellen. Es soll auch den Zweck erfüllen, sich mit der therapeutischen Anwendung von Lokaltherapien wie auch Lasertherapien $\mathrm{zu}$ befassen und $\mathrm{zu}$ erkennen, dass es eine Fülle von medizinischen Indikationen wie auch Therapiemethoden gibt, die nicht nur als kosmetisch $\mathrm{zu}$ bewerten sind. Dazu bedarf es allerdings genauer Kenntnis und Erfahrung in der jeweiligen Behandlungsmethode, um die jeweils am besten geeignete Therapie zu bestimmen. Auch von Bedeutung ist die Tatsache, dass selbst genetisch bedingte Störungen durchaus gut einer Therapie zugänglich sein können, andere wiederum kaum oder zumindest mit einer hohen Zahl von Rezidiven oder möglichen Nebenwirkungen belastet sein können. So stellt sich bei Chemical Peels die Frage, welche Substanz in welcher Konzentration auszuwählen ist, und für die geeignete Laserbehandlung muss abgewogen werden, welche Wellenlänge, Spotgröße, Pulsdauer und Energie geeignet sind, und letztlich, ob man nicht doch mit lokalen Behandlungen einen Erfolg erreichen könnte oder gar dem Patienten generell von einer Behandlung abrät.
K. Fritz
C. Salavastru

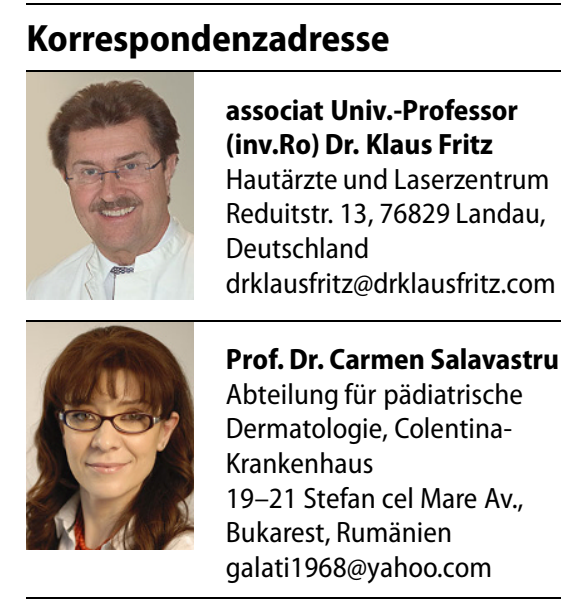

Interessenkonflikt. K. Fritz und C. Salavastru geben an, dass kein Interessenkonflikt besteht. 